\title{
TWO PROBLEMS ON POTENTIAL THEORY FOR UNBOUNDED SETS
}

\author{
U. CEGRELL, S. KOLODZIEJ* AND N. LEVENBERG
}

\section{Introduction. Statement of the problems.}

We consider two problems which are well-known in classical potential theory when one studies bounded sets and measures having compact support. Let $L$ denote the plurisubharmonic (psh) functions in $\mathrm{C}^{N}$ of logarithmic growth:

$$
L=\left\{u \operatorname{psh} \text { in } \mathrm{C}^{N}: u(z)-\log |z| \leq c=c(u),|z| \rightarrow+\infty\right\} .
$$

This class, as well as associated subclasses, have been extensively studied in relation with extremal psh functions. For an arbitrary Borel set $E \subset \mathrm{C}^{N}$ we define the $L$-extremal function

$$
V_{E}(z)=\sup \{u(z): u \in L, u \leq 0 \text { on } E\}
$$

and $V_{E}^{*}(z):=\limsup _{\zeta \rightarrow z} V_{E}(\zeta)$. If $V_{E}^{*} \not \equiv 0,+\infty$, then $V_{E}^{*} \in L$ and $\left\{z \in E: V_{E}^{*}(z)>0\right\}$ is pluripolar. Recall that a set $E$ is pluripolar (equivalently, $\left.V_{E}^{*} \equiv+\infty\right)$ if there exists a psh function $u$ with $E \subset\{z: u(z)=-\infty\}$; in this case, we can even take $u \in L$. Let $E_{j}, j=1,2, \ldots$ be an increasing sequence of Borel sets in $\mathrm{C}^{N}$ and let $E:=\cup E_{j}$. If $E$ is bounded (or if $E$ is pluripolar), then

(0.1) $\lim _{j \rightarrow+\infty} V_{E_{j}}^{*}=V_{E}^{*}$ pointwise on $\mathrm{C}^{N}$.

Here's a quick proof of (0.1) (see [5]). Clearly if $E$ is pluripolar, so is each $E_{j}$ and $V_{E_{j}}^{*}=V_{E}^{*} \equiv+\infty$. Suppose $E$ is not pluripolar. Clearly $V:=\lim _{j \rightarrow+\infty} V_{E_{j}}^{*} \geq V_{E}^{*}$ and $V \in L$. Set

$$
F:=\cup_{j}\left\{z \in E_{j}: V_{E_{j}}^{*}(z)>0\right\} ;
$$

then $F$ is pluripolar and $V=0$ on $E-F$. Hence $V \leq V_{E-F}^{*}$ and we are done provided we can show:

(0.2) if a set $F$ is pluripolar, then $V_{E}^{*}=V_{E-F}^{*}$.

Now clearly $V_{E}^{*} \leq V_{E-F}^{*}$. For the reverse inequality, we can find $v \in L$ with

*Partially supported by KBN Grant No. 2 PO3A 05809

Received July 22, 1996. 
$F \subset\{z: v(z)=-\infty\}$; since $E-F$ is bounded and $v$ is uppersemicontinuous we can assume $v \leq 0$ on $E-F$. Then for any $u \in L$ with $u \leq 0$ on $E-F$ and any $\epsilon>0$,

$$
(1-\epsilon) u+\epsilon v \leq V_{E} \leq V_{E}^{*}
$$

Thus $u \leq V_{E}^{*}$ almost everywhere (a. e.) and hence everywhere.

Note how boundedness of $E$ was used. If $E$ is unbounded, we show that (0.1) and (0.2) are still true if $N=1$ (Theorem 1.2 and Corollary 1.4) but not if $N>1$. This was a problem posed by Plesniak.

The other result is a positivity and uniqueness theorem for the logarithmic energy

$$
I(\mu, \mu):=\iint \log \frac{1}{|x-y|} d \mu(x) d \mu(y)
$$

of signed Borel measures in $\mathrm{R}^{n}$ which have total mass 0 but which are not necessarily compactly supported. We recall the classical result.

Positivity And Uniqueness Lemma ([6], Theorem 1.16)(n=2). If $\mu$ is a signed Borel measure in $\mathrm{R}^{2}$ with $I(|\mu|,|\mu|)<+\infty$ and $|\mu|(1)<+\infty$ having compact support and either $\mu(1)=0$ or the support of $\mu$ is contained inside the unit disk, then $I(\mu, \mu) \geq 0$ and $I(\mu, \mu)=0$ if and only if $\mu=0$.

The lemma can be extended to certain non-compactly supported measures in $\mathrm{R}^{n}$ for any $n \geq 2$ (Theorem 2.5). Our first attempt at solving the Plesniak problem in $\mathrm{C}=\mathrm{R}^{2}$ led us to this result.

In the case $N=1$, the class $L$, also called the class of subharmonic functions of minimal growth, has been studied by many authors including Arsove, Essén, Haliste, Hayman, Huber, Lewis, Shea and $\mathrm{Wu}$; we refer the reader to [3] for specific references.

We would like to thank the mathematics departments of Umea University and the University of Auckland as well as the New Zealand Mathematical Society for their support. The third author would also like to thank V. Totik for many helpful discussions. In addition, the authors thank the referee for valuable comments.

\section{The Plesniak problem.}

The Plesniak problem (both (0.1) and (0.2)) has a negative answer in $C^{2}$ as the following example due to [2] shows.

ExAmple 1.1. Let $E=\left\{\left(z_{1}, z_{2}\right) \in \mathrm{C}^{2}:\left|z_{2}\right| \leq 1\right\} \cup\left\{\left(z_{1}, z_{2}\right) \in \mathrm{C}^{2}: z_{1}=0\right.$, $\left.\left|z_{2}\right| \leq 2\right\}$ and $F=\left\{\left(z_{1}, z_{2}\right) \in \mathrm{C}^{2}: z_{1}=0,1<\left|z_{2}\right| \leq 2\right\}$. Then 


$$
V_{E-F}^{*}\left(z_{1}, z_{2}\right)=\log ^{+}\left|z_{2}\right| \neq \log ^{+} \frac{\left|z_{2}\right|}{2}=V_{E}^{*}\left(z_{1}, z_{2}\right) .
$$

Proof. Clearly $V_{E-F}^{*}\left(z_{1}, z_{2}\right)=\log ^{+}\left|z_{2}\right|$. To see that $V_{E}^{*}\left(z_{1}, z_{2}\right)=\log ^{+} \frac{\left|z_{2}\right|}{2}$, note first that since $E \subset\left\{\left(z_{1}, z_{2}\right) \in \mathrm{C}^{2}:\left|z_{2}\right| \leq 2\right\}$, we have $V_{E}^{*}\left(z_{1}, z_{2}\right)$ $\geq V_{E}\left(z_{1}, z_{2}\right) \geq \log ^{+} \frac{\left|z_{2}\right|}{2}$. Now, since $E-F \subset E, \quad V_{E}\left(z_{1}, z_{2}\right) \leq V_{E}^{*}\left(z_{1}, z_{2}\right)$ $\leq \log ^{+}\left|z_{2}\right|$. This shows that $V_{E}, V_{E}^{*}$ are independent of $z_{1}$; for if we fix $z_{2}^{0}$, then $f\left(z_{1}\right):=V_{E}^{*}\left(z_{1}, z_{2}^{0}\right) \leq \log ^{+}\left|z_{2}^{0}\right|$ for all $z_{1}$; thus $f$ is bounded and hence constant. Since $V_{E}\left(0, z_{2}\right)=0$ for $\left|z_{2}\right| \leq 2$ we have $V_{E}\left(0, z_{2}\right) \leq \log ^{+} \frac{\left|z_{2}\right|}{2}$; thus this better bound persists on all of $\mathrm{C}^{2}$; i.e., $V_{E}\left(z_{1}, z_{2}\right)=\log ^{+} \frac{\left|z_{2}\right|}{2}$.

Note, in this example, for any sequence of bounded Borel sets $E_{j}$ with $E_{j} \subset E_{j+1}, E=\cup E_{j}$, we have

$$
\lim _{j \rightarrow+\infty} V_{E_{j}}^{*}\left(z_{1}, z_{2}\right)=V_{E-F}^{*}\left(z_{1}, z_{2}\right)=\log ^{+}\left|z_{2}\right| \neq V_{E}^{*}\left(z_{1}, z_{2}\right) .
$$

We see here the phenomenon of propagation of singularities that can occur in $\mathrm{C}^{N}$ for $N>1$ but not in $\mathrm{C}$; the one-variable proof sketched in the introduction of equality of $V_{E-F}^{*}$ and $V_{E}^{*}$ in the bounded case fails for the following reason: if $u \in L$ and $u=-\infty$ on $F$, then necessarily $u=-\infty$ on the whole complex line

$$
F^{*}:=\left\{\left(z_{1}, z_{2}\right) \in \mathrm{C}^{2}: z_{1}=0, z_{2} \in \mathrm{C}\right\}
$$

( $F^{*}$ is the pluripolar hull of $F$; see [1] for further results). For any $0<\alpha \leq 1$, the function $v_{\alpha}(z):=\alpha \log \left|z_{1}\right|$ is $-\infty$ precisely on $F^{*}$; clearly these functions, and hence any $v \in L$ which is $-\infty$ on $F^{*}$, cannot remain bounded above on $E-F$.

THEOREM 1.2. Let $E_{j}, j=1,2, \ldots$ be an increasing sequence of Borel sets in $\mathrm{C}$ and let $E:=\cup E_{j}$. Then

$$
\lim _{j \rightarrow+\infty} V_{E_{j}}^{*}=V_{E}^{*}
$$

Proof. The proof is based on the following preliminary result. Below, "q.e. on $E$ " means everywhere on $E$ except perhaps a polar set; $\mathrm{SH}(U)$ denotes the subharmonic functions on $U$; and " $\phi \leq M$ on $\partial U$ " means $\lim \sup _{z \rightarrow \zeta} \phi(z) \leq M$ for all $\zeta \in \partial U$.

Lemma 1.3. Let $u \in L, u \geq 0$ (but $u \neq \equiv$ ) and $E \subset \mathrm{C}$ with $u=0$ q.e. on $E$. Let $U$ be a connected component of $\{u<M\}$ for some $M>0$. Suppose that

$$
u_{\mid U}=\left[\sup \left\{\phi \in \mathrm{SH}(U): \phi \leq 0 \text { on } E^{\prime}, \phi \leq M \text { on } \partial U\right\}\right]^{*}
$$

where $E^{\prime}:=\{u=0\} \cap U$. Then given $c>0$ with $c<M$ and given $z_{0} \in U-E$, there exists $w \in L$ such that 
1. $w=u$ in the complement of $U$;

2. $w \leq c$ on $E \cap U$;

3. $w \leq u+c$ everywhere in $\mathrm{C}$; and

4. $w\left(z_{0}\right)>u\left(z_{0}\right)-c$.

Furthermore, if $F^{\prime}:=\{w=c\} \cap U$, then

$$
w_{\mid U}=\left[\sup \left\{\phi \in \operatorname{SH}(U): \phi \leq c \text { on } F^{\prime}, \phi \leq M \text { on } \partial U\right\}\right]^{*} .
$$

Proof of Lemma 1.3. The set $U$ is bounded by the theorem of WimanValiron (see [3], page 384 or [4], page 197). For this theorem tells us that given $t>1$, there exist $R_{j} \uparrow+\infty$ such that

$$
\inf _{|z|=R_{j}} u(z)>\frac{1}{t} \sup _{|z|=R_{j}} u(z) .
$$

Clearly $\lim _{j \rightarrow+\infty} \sup _{|z|=R_{j}} u(z)=+\infty$; hence, given $M>0$, we can choose $R_{j}$ with $U \cap\left\{z:|z|<R_{j}\right\} \neq \emptyset$ and

$$
\inf _{|z|=R_{j}} u(z)>M
$$

Since $U$ is connected, it is path connected; using this, an easy exercise shows that $U \subset\left\{z:|z|<R_{j}\right\}$.

Recall we are assuming that

$$
u_{\mid U}=\left[\sup \left\{\phi \in \operatorname{SH}(U): \phi \leq 0 \text { on } E^{\prime}, \phi \leq M \text { on } \partial U\right\}\right]^{*}
$$

where $E^{\prime}:=\{u=0\} \cap U$. Now since $E \backslash E^{\prime}$ is polar, we can find $g$ subharmonic in $\mathrm{C}$ with $g \leq 0$ on $U$ and $E \backslash E^{\prime} \subset\{g=-\infty\}$. We let $U_{j} \uparrow U, j=0,1,2, \ldots$ be an exhaustion sequence for $U$ with the property that $g$ is bounded on the boundary of every $U_{j}$. This can be achieved since by Theorem 6.3 in [3], given $z_{0} \in \mathrm{C}$ and $R>0$, one can find a circle $\left\{z:\left|z-z_{0}\right|=r\right\}, r<R$, on which $g$ is bounded. Then any compact set contained in $U$ may be covered by the interiors of these circles.

Fix a sequence of positive numbers $\left\{c_{j}\right\}$ with $c_{j}<c_{j+1} \uparrow c$. Set

$$
h_{1}:=\left[\sup \left\{\phi \in \operatorname{SH}(U): \phi \leq c_{1} \text { on } E^{\prime}, \phi \leq M \text { on } \partial U\right\}\right]^{*} .
$$

Then $u<h_{1} \leq u+c_{1}$ in $U$. In particular,

$$
h_{1}(x)=c_{1} \text { for } x \in E^{\prime} .
$$

Since $h_{1}>u$ in $U$ and $g$ is bounded on $\partial U_{0} \subset \subset U$, we can choose $d_{1}>0$ such that $h_{1}+d_{1} g>u$ on $\partial U_{0}$ and

$$
d_{1} g\left(z_{0}\right)>-c_{1} .
$$

Then 


$$
f_{1}:= \begin{cases}h_{1}+d_{1} g, & \text { on } U_{0} \\ \max \left(h_{1}+d_{1} g, u\right), & \text { on } U \backslash U_{0} \\ u, & \text { on } \mathrm{C} \backslash U\end{cases}
$$

is a well defined, subharmonic function. We have $f_{1}\left(z_{0}\right)>u\left(z_{0}\right)-c_{1}$ and $f_{1} \leq c_{1}$ on $E^{\prime}$ (see (1.2)). Furthermore, since $g$ is negative and $c_{1}$ is positive,

$$
h_{1}+d_{1} g \leq h_{1} \leq u+c_{1}
$$

which shows that

$$
f_{1} \leq u+c_{1}
$$

Note $E^{\prime} \subset E_{1}^{\prime}:=\left\{f_{1} \leq c_{1}\right\}$ from (1.4). Also, since $f_{1}=-\infty$ on $\left(E-E^{\prime}\right) \cap U_{0}$, we have

$$
\left(E-E^{\prime}\right) \cap U_{0} \subset \text { int } E_{1}^{\prime}
$$

Set

$$
u_{1}:= \begin{cases}{\left[\sup \left\{\phi \in \mathrm{SH}(U): \phi \leq c_{1} \text { on } E_{1}^{\prime}, \phi \leq M \text { on } \partial U\right\}\right]^{*},} & \text { on } U \\ u, & \text { on } \mathrm{C} \backslash U .\end{cases}
$$

Since $E^{\prime} \subset E_{1}^{\prime}$, if $\phi \leq c_{1}$ on $E_{1}^{\prime}$ we have $\phi \leq c_{1}$ on $E^{\prime}$ which shows that $u_{1} \leq h_{1}$. Thus, for $z \in E^{\prime}$ we have $u_{1}(z) \leq h_{1}(z) \leq u(z)+c_{1}=c_{1}$ which means that $z \in E_{1}:=\left\{u_{1} \leq c_{1}\right\}$; i.e., $E^{\prime} \subset E_{1}$. We claim that, together with (1.5), this shows that

$$
E^{\prime} \cup\left[\left(E-E^{\prime}\right) \cap U_{0}\right] \subset E_{1} .
$$

For if int $E_{1}^{\prime}=\emptyset$, there is nothing to prove. If, on the other hand, int $E_{1}^{\prime} \neq \emptyset$, then $u_{1}=c_{1}$ on int $E_{1}^{\prime}$ which shows that int $E_{1}^{\prime} \subset E_{1}$. Moreover, as noted above, $u_{1} \leq u+c_{1}$; also, from (1.3), $u_{1}\left(z_{0}\right) \geq u\left(z_{0}\right)-c_{1}$. Thus we have a subharmonic function $u_{1} \in L$ having the following properties:

$$
\begin{aligned}
& u_{1} \leq u+c_{1} ; \\
& E^{\prime} \cup\left[U_{0} \cap\left(E-E^{\prime}\right)\right] \subset E_{1}:=\left\{u_{1} \leq c_{1}\right\} \\
& u_{1}=u \text { outside } U ; \text { and } \\
& u_{1}\left(z_{0}\right) \geq u\left(z_{0}\right)-c_{1} .
\end{aligned}
$$

If we repeat this construction with $u_{1}, U_{1}, E_{1}$ and $c_{2}-c_{1}$ in place of $u, U_{0}, E^{\prime}$ and $c_{1}$, we obtain $u_{2} \in L$ having the following properties:

$$
\begin{aligned}
& u_{2} \leq u_{1}+\left(c_{2}-c_{1}\right) ; \\
& E^{\prime} \cup\left[U_{1} \cap\left(E-E^{\prime}\right)\right] \subset E_{1} \cup\left[U_{1} \cap\left(E-E_{1}\right)\right] \subset E_{2}:=\left\{u_{2} \leq c_{2}\right\} \\
& u_{2}=u \text { outside } U ; \text { and } \\
& u_{2}\left(z_{0}\right) \geq u_{1}\left(z_{0}\right)-\left(c_{2}-c_{1}\right) \geq u\left(z_{0}\right)-c_{1}-\left(c_{2}-c_{1}\right)=u\left(z_{0}\right)-c_{2} .
\end{aligned}
$$

We continue in this fashion getting a sequence $\left\{u_{j}\right\}$ such that $u_{j}-c_{j}$ is decreasing; it is easy to see that the function $w:=\lim u_{j}$ has all the required properties. 
Proof of Theorem 1.2. Let $E_{j}, j=1,2, \ldots$ be an increasing sequence of Borel sets in $\mathrm{C}^{N}$ with $E:=\cup E_{j}$ and let $V:=\lim _{j \rightarrow \infty} V_{E_{j}}^{*}$. Then $V \geq V_{E}^{*}$. If $V \equiv 0$, then $V \geq V_{E}^{*}$ implies $V_{E}^{*} \equiv 0$. Since the case where $V \equiv+\infty$ was covered in the introduction, we need only consider the case where $V \not \equiv 0,+\infty$; i.e., $V \in L$, and it clearly suffices to show: given $c>0$ and $z_{0} \in \mathrm{C}-E$, there exists $\psi \in L$ with $\psi \leq c$ on $E$ and $\psi\left(z_{0}\right)>V\left(z_{0}\right)-c$. For then $\psi-c \leq 0$ on $E$; hence $V_{E}^{*}\left(z_{0}\right) \geq \psi\left(z_{0}\right)-c>V\left(z_{0}\right)-2 c$; this inequality being valid for all $c>0$ yields the result. To prove the italicised statement, note first that $V$ satisfies the hypothesis (1.1) of Lemma 1.3. We now fix an exhaustion sequence for $\mathrm{C}$ of open sets $U_{j}$, each of them being a connected component of $\left\{V<M_{j}\right\}$ containing $z_{0}$; here, the sequence $\left\{M_{j}\right\}$ is chosen with $M_{j} \rightarrow \infty$. Fix $\left\{c_{j}\right\}$ with $c_{0}=0, c_{j}>0$ for $j>0$, and $\sum c_{j}<c$. We apply the lemma repeatedly, first with the data $\left(V, c_{0}, U_{0}\right)$, obtaining $u_{1}$ as the new function; then with data $\left(u_{1}, c_{1}, U_{1}\right)$ to get $u_{2}$; etc. Since $u_{j}-\sum_{k=0}^{j-1} c_{j}$ is decreasing and $\sum c_{j}<c$, the limit $\psi=\lim u_{j}$ exists; $\psi \in L$; and, by the construction, $\psi$ satisfies the required inequalities.

Corollary 1.4. Suppose $E$ is a Borel set in $\mathrm{C}$ and $F$ is a polar set. Then $V_{E-F}^{*}=V_{E}^{*}$.

Proof. Take $E_{j}$ bounded with $E_{j} \uparrow E$. Then

$$
V_{E-F}^{*}=\lim _{j \rightarrow \infty} V_{E_{j}-F}^{*}=\lim _{j \rightarrow \infty} V_{E_{j}}^{*}=V_{E}^{*} .
$$

Remark. As pointed out to the third author by S. Gardiner, for unbounded $E, V_{E}^{*} \in L$ (i.e., $V_{E}^{*} \not \equiv 0,+\infty$ ) if and only if $E$ is not polar and $E^{*}:=\{z: 1 / z \in E\}$ is thin at 0 . In this case, the total mass $\mu(1)$ of the Laplacian of $V_{E}^{*}$ equals $2 \pi$; indeed, a characterization of this subclass of the class $L$ can be found in [3], Theorem 6.32: for a function $u \in L, \mu(1)=2 \pi$ if and only if

$$
\lim _{r \rightarrow+\infty} \frac{\sup _{|z|=r} u(z)}{\log r}=1
$$

\section{Logarithmic potentials in $\mathrm{R}^{n}$.}

In this section, we discuss some general results about logarithmic potentials and energies of measures in $\mathrm{R}^{n}$ for any $n=2,3, \ldots$. Let $\mu$ be a positive measure on $\mathrm{R}^{n}$ satisfying

$$
\int \log (1+|t|) d \mu(t)<+\infty .
$$

Then the logarithmic potential 


$$
p_{\mu}(x)=\int \log \frac{1}{|x-y|} d \mu(y)
$$

is locally integrable and superharmonic in all of $\mathrm{R}^{n}$. Furthermore, since

$$
\log \frac{1}{|x-y|} \geq-[\log (1+|x|)+\log (1+|y|)],
$$

(2.1) implies that

$$
\int p_{\mu}(x) d \mu(x)=\int\left[\int \log \frac{1}{|x-y|} d \mu(y)\right] d \mu(x)>-\infty .
$$

Definition. Let $\mu$ and $\nu$ be two positive measures satisfying (2.1). We call

$$
I(\mu, \nu)=\int\left[\int \log \frac{1}{|x-y|} d \mu(x)\right] d \nu(y):=\lim _{M \rightarrow+\infty} \int\left[\int \min \left(\log \frac{1}{|x-y|}, M\right) d \mu(x)\right] d \nu(y)
$$

the mutual energy of $\mu$ and $\nu$.

Note that by Fubini's theorem we have $I(\mu, \nu)=I(\nu, \mu)$. Also, $I(\mu, \nu)$ may be equal to $+\infty$. Also note that again (2.1) implies that $I(\mu, \nu)>-\infty$. When is $I(\mu, \nu)<+\infty$ ?

LeMma 2.1. Let $\mu, \nu$ be two positive measures satisfying

$$
\int \log (1+|t|) d(\mu+\nu)(t)<+\infty
$$

Then $p_{\mu}(x):=\int \log \frac{1}{|x-y|} d \mu(y) \in L^{1}(d \nu)$ if and only if $I(\mu, \nu)<+\infty$. In this case,

$$
\lim _{R, S \rightarrow+\infty} I\left(\chi_{B(0, R)} \mu, \chi_{B(0, S)} \nu\right)=I(\mu, \nu)
$$

where $\chi_{B(0, R)}$ denotes the characteristic function of the ball $B(0, R)=$ $\{x:|x|<R\}$.

Proof. Clearly if $p_{\mu} \in L^{1}(d \nu)$ then $I(\mu, \nu)<+\infty$. For the converse, suppose $I(\mu, \nu)<+\infty$. For $M \geq 0$, we have

$$
\log \frac{1}{|x-y|} \geq \min \left(\log \frac{1}{|x-y|}, M\right) \geq-[\log (1+|x|)+\log (1+|y|)] .
$$

Now

$$
\begin{aligned}
& p_{\mu}(x)=\int\left[\log \frac{1}{|x-y|}+\log (1+|x|)+\log (1+|y|)\right] d \mu(y)-\mu(1)[\log (1+|x|)] \\
& -\int \log (1+|y|) d \mu(y):=f_{1}(x)-f_{2}(x)
\end{aligned}
$$


where $f_{2}(x):=\mu(1)[\log (1+|x|)]+\int \log (1+|y|) d \mu(y) \in L^{1}(d \nu)$ by hypothesis (2.3). Since

$$
f_{1}(x):=\int\left[\log \frac{1}{|x-y|}+\log (1+|x|)+\log (1+|y|)\right] d \mu(y) \geq 0,
$$

it follows that $f_{1} \in L^{1}(d \nu)$ if

$$
\lim _{M \rightarrow+\infty} \int\left\{\int\left[\min \left(\log \frac{1}{|x-y|}, M\right)+\log (1+|x|)+\log (1+|y|)\right] d \mu(y)\right\} d \nu(x)<+\infty .
$$

This inequality follows from (2.3), the definition (2.2), and the assumption that $I(\mu, \nu)<+\infty$.

Finally for each $M \geq 0$, the equality

$$
\begin{gathered}
\lim _{R, S \rightarrow+\infty} \int\left[\int \min \left(\log \frac{1}{|x-y|}, M\right) d\left[\chi_{B(0, R)} \mu\right](x)\right] d\left[\chi_{B(0, S)} \nu\right](y)= \\
\int\left[\int \min \left(\log \frac{1}{|x-y|}, M\right) d \mu(x)\right] d \nu(y)
\end{gathered}
$$

follows from the monotone convergence theorem. Thus under the assumption $I(\mu, \nu)<+\infty$,

$$
\begin{gathered}
I(\mu, \nu)=\lim _{M, R, S \rightarrow+\infty} \int\left[\int \min \left(\log \frac{1}{|x-y|}, M\right) d\left[\chi_{B(0, R)} \mu\right](x)\right] d\left[\chi_{B(0, S)} \nu\right](y) \\
=\lim _{R, S \rightarrow+\infty} I\left(\chi_{B(0, R)} \mu, \chi_{B(0, S)} \nu\right) .
\end{gathered}
$$

REMARK. Lemma 2.1 says that under assumption (2.3),

$$
p_{\mu} \in L^{1}(d \nu) \text { if and only if }|\log | x-y|| \in L^{1}(\mu \times \nu) .
$$

We next turn to the case of signed Borel measures.

Definition. Suppose $\mu$ is a signed Borel measure satisfying

$$
\int \log (1+|y|) d|\mu|(y)<+\infty .
$$

We define the logarithmic potential

$$
p_{\mu}(x)=\int \log \frac{1}{|x-y|} d \mu(y) .
$$

If $\nu$ is another signed Borel measure satisfying

$$
\int \log (1+|y|) d|\nu|(y)<+\infty,
$$


and we have $I(|\mu|,|\nu|)<+\infty$, we define the mutual energy of $\mu, \nu$ by

$$
I(\mu, \nu):=\iint \log \frac{1}{|x-y|} d \mu(x) d \nu(y) .
$$

The following result, whose proof is similar to that of Lemma 2.1, shows that this is a good definition.

Corollary 2.2. Let $\mu, \nu$ be two signed Borel measures satisfying (2.4), $\left(2.4^{\prime}\right)$ and $I(|\mu|,|\nu|)<+\infty$. Then

1. $p_{\mu} \in L^{1}(\nu), p_{\nu} \in L^{1}(\mu)$;

2. $|I(\mu, \nu)|<+\infty$;

3. $I(\mu, \nu)=I(\nu, \mu)$;

4. $\lim _{R, S \rightarrow+\infty} I\left(\chi_{B(0, R)} \mu, \chi_{B(0, S)} \nu\right)=I(\mu, \nu)$.

In particular, we get finiteness of the energy $I(\mu, \mu)$ for a signed Borel measure satisfying (2.4) and $I(|\mu|,|\mu|)<+\infty$. Under an extra hypothesis, the energy is nonnegative. We first deal with some preliminaries. Below, we let $d m=d m(x)$ denote Lebesgue measure on $\mathrm{R}^{n}$; we let $D=D\left(\mathrm{R}^{n}\right)$ denote the space of real-valued smooth $\left(C^{\infty}\right)$ functions on $\mathrm{R}^{n}$ of compact support; we let $S$ be the space of rapidly decreasing functions on $\mathrm{R}^{n}$; and, finally, $S^{\prime}$ denotes the space of tempered distributions.

Lemma 2.3. Let $0<a<n$. For $f \in D$,

$$
C_{a} \iint \frac{f(x) f(y)}{|x-y|^{n-a}} d m(x) d m(y)=\int \frac{|\hat{f}(\xi)|^{2}}{|\xi|^{a}} d m(\xi)
$$

where $C_{a}=\pi^{a-n / 2} \frac{\Gamma\left(\frac{n-a}{2}\right)}{\Gamma\left(\frac{a}{2}\right)}>0$ and $\hat{f}(\xi):=\int f(x) e^{-2 \pi i x \cdot \xi} d m(x)$ (here, $x \cdot \xi:=$ $\left.\sum_{i=1}^{n} x_{i} \xi_{i}\right)$.

Proof. Let $\phi(x):=\exp \left(-\pi|x|^{2}\right)$. Then $\phi=\hat{\phi}$. Also,

$$
\frac{\widehat{C_{a}}}{|x|^{n-a}}=\frac{1}{|\xi|^{a}} \text { for } 0<a<n / 2 ;
$$

and, by the convolution identity $\frac{C_{a}}{|x|^{n-a}}=\frac{C_{a / 2}}{|x|^{n-a / 2}} * \frac{C_{a / 2}}{|x|^{n-a / 2}}$ for $0<a<n / 2$, the above equation holds for $0<a<n$. For $t>0$, let $\phi_{t}(x)=\phi(x / t)$. Then $\hat{\phi}_{t}(\xi)=t^{n} \hat{\phi}(t \xi)=t^{n} \phi(t \xi)$ and hence

$$
\frac{1}{|\xi|^{a}} * \hat{\phi}_{t}(\xi)=\frac{1}{|\xi|^{a}} * t^{n} \phi(t \xi) \rightarrow \frac{1}{|\xi|^{a}}
$$

as $t \rightarrow+\infty$. Note also that

$$
\phi_{t}(x) \rightarrow 1
$$

as $t \rightarrow+\infty$. We show that 


$$
\int\left[\int \frac{\phi_{t}(x-y)}{|x-y|^{n-a}} f(y) d m(y)\right] f(x) d m(x)=\int \frac{\widehat{\phi_{t}(x)}}{|x|^{n-a}}(\xi)|\hat{f}(\xi)|^{2} d m(\xi) .
$$

Then since

$$
C_{a} \frac{\widehat{\phi_{t}(x)}}{|x|^{n-a}}(\xi)=\hat{\phi}_{t}(\xi) *\left[\frac{\widehat{C_{a}}}{|x|^{n-a}}\right](\xi)=t^{n} \phi(t \xi) * \frac{1}{|\xi|^{a}} \rightarrow \frac{1}{|\xi|^{a}}
$$

as $t \rightarrow+\infty$ by (2.6), equations (2.7) and (2.8) prove the lemma.

To prove (2.8), we use Parseval's identity:

$$
\begin{gathered}
\int\left[\int \frac{\phi_{t}(x-y)}{|x-y|^{n-a}} f(y) d m(y)\right] f(x) d m(x)=\int\left[\int \frac{\phi_{t}(x-\widehat{y)}}{|x-y|^{n-a}} f(y) d m(y)\right](\xi) \overline{\hat{f}(\xi)} d m(\xi) \\
=\int \frac{\widehat{\phi_{t}(x)}}{|x|^{n-a}}(\xi)|\hat{f}(\xi)|^{2} d m(\xi) .
\end{gathered}
$$

Lemma 2.4. For $f \in D$ with $\int f d m=0$,

$$
I(f, f):=\iint \log \frac{1}{|x-y|} f(x) f(y) d m(x) d m(y)=d_{n} \int \frac{|\hat{f}(\xi)|^{2}}{|\xi|^{n}} d m(\xi)
$$

where $d_{n}:=2\left[\frac{\pi^{n / 2}}{\Gamma\left(\frac{2}{2}\right)}\right]$.

Proof. We have

$$
\begin{gathered}
C_{a} \int \frac{f(x)}{|y-x|^{n-a}} d m(x)=C_{a} \int f(x)\left[\frac{1}{|y-x|^{n-a}}-1\right] d m(x) \\
=(n-a) C_{a} \int f(x)\left[\frac{1}{\frac{|y-x|^{n-a}}{n-a}}\right] d m(x) \rightarrow d_{n} \int f(x) \log \frac{1}{|x-y|} d m(x) \text { as } a \rightarrow n
\end{gathered}
$$

since $(n-a) C_{a} \rightarrow d_{n}$ as $a \rightarrow n$. Now use Lemma 2.3. Note that the fact that $\hat{f}(0)=0$ shows that $\frac{|\hat{f}(\xi)|^{2}}{|\xi|^{n}}$ is integrable near $\xi=0$.

THEOREM 2.5. Let $\nu$ be a signed measure satisfying

1. $\int \log (1+|t|) d|\nu|(t)<+\infty$;

2. $\nu(1)=0$;

3. $I(|\nu|,|\nu|)<+\infty$.

Then $I(\nu, \nu) \geq 0$ with equality if and only if $\nu=0$.

Proof. Note from 1., 3., and Corollary 2.2 we get $I(\nu, \nu)<+\infty$.

We first give the proof if $\nu$ is assumed to have compact support. Let 
$\nu=\nu^{+}-\nu^{-}$be the Jordan decomposition of $\nu$. Given a standard smoothing kernel $\quad \psi \in D \quad\left(\psi \geq 0, \int \psi(x) d m(x)=1, \psi(x)=0 \quad\right.$ if $\left.\quad|x| \geq 1\right)$, we let $\psi_{\epsilon}(x)=\frac{1}{\epsilon^{n}} \psi(x / \epsilon)$ and $\nu_{\epsilon}=\psi_{\epsilon} * \nu$. Then $\nu_{\epsilon} \in D$ and $\int \nu_{\epsilon}(x) d m(x)=0$. By Lemma 2.4,

$$
I\left(\nu_{\epsilon}, \nu_{\epsilon}\right)=d_{n} \int \frac{\left|\hat{\nu}_{\epsilon}(\xi)\right|^{2}}{|\xi|^{n}} d m(\xi) \geq 0
$$

We show that

$$
\lim _{\epsilon \rightarrow 0} I\left(\nu_{\epsilon}, \nu_{\epsilon}\right)=I(\nu, \nu) .
$$

First of all, we note that $p_{\nu^{+}}(x):=\int \log \frac{1}{|x-y|} d \nu^{+}(y)$ is superharmonic so that

$$
p_{\nu_{\epsilon}^{+}}(x):=\int \log \frac{1}{|x-y|} d \nu_{\epsilon}^{+}(y) \rightarrow p_{\nu^{+}}(x)
$$

pointwise on $\mathbf{R}^{n}$. Thus, since $\nu_{\eta} \rightarrow \nu$ weak- $*$ as $\eta \rightarrow 0$, for each $\epsilon>0$,

$$
\int p_{\nu_{\epsilon}^{+}}(x) d \nu_{\eta}^{+}(x) \rightarrow \int p_{\nu_{\epsilon}^{+}}(x) d \nu^{+}(x) .
$$

By 3. and dominated convergence, we have

$$
\int p_{\nu_{\epsilon}^{+}}(x) d \nu_{\eta}^{+}(x) \rightarrow \int p_{\nu^{+}}(x) d \nu^{+}(x)
$$

as $\epsilon, \eta \rightarrow 0$. Similarly,

$$
\int p_{\nu_{\epsilon}^{-}}(x) d \nu_{\eta}^{-}(x) \rightarrow \int p_{\nu^{-}}(x) d \nu^{-}(x)
$$

and

$$
\int p_{\nu_{\epsilon}^{+}}(x) d \nu_{\eta}^{-}(x) \rightarrow \int p_{\nu^{+}}(x) d \nu^{-}(x)
$$

as $\epsilon, \eta \rightarrow 0$. This proves (2.10). Together with (2.9), this shows that

$$
I(\nu, \nu) \geq 0
$$

in the case where $\nu$ has compact support. To prove the uniqueness assertion in this case, since $\nu, \nu_{\epsilon}$ have compact support, if

$$
I\left(\nu_{\epsilon}, \nu_{\epsilon}\right)=d_{n} \int \frac{\left|\hat{\nu}_{\epsilon}(\xi)\right|^{2}}{|\xi|^{n}} d m(\xi)=d_{n} \int \frac{\left|\widehat{\psi_{\epsilon} * \nu}(\xi)\right|^{2}}{|\xi|^{n}} d m(\xi) \rightarrow 0
$$

then $\widehat{\psi_{\epsilon} * \nu} \rightarrow 0$ in $L^{2}\left(\mathrm{R}^{n}\right)$. By Parseval's theorem, it follows that $\psi_{\epsilon} * \nu \rightarrow 0$ in $L^{2}\left(\mathrm{R}^{n}\right)$ and hence $\nu=0$. 
If $\nu$ is not assumed to have compact support, consider

$$
\nu_{R}:=\chi_{B(0, R)} \nu-\nu(B(0, R)) \sigma
$$

where $d \sigma=\frac{d m}{m(B(0,1))}$ denotes normalized Lebesgue measure on the unit ball. Then $\nu_{R}(1)=0$ so that from the previous case we have

$$
I\left(\nu_{R}, \nu_{R}\right) \geq 0 \text {. }
$$

Using Corollary 2.2 and hypothesis 2 ., as $R \nearrow+\infty$,

$$
\begin{gathered}
I\left(\chi_{B(0, R)} \nu, \chi_{B(0, R)} \nu\right) \rightarrow I(\nu, \nu), \\
I\left(\chi_{B(0, R)} \nu, \nu(B(0, R)) \sigma\right) \rightarrow I(\nu, \nu(1) \sigma)=0 \text { and } \\
I(\nu(B(0, R)) \sigma, \nu(B(0, R)) \sigma) \rightarrow I(\nu(1) \sigma, \nu(1) \sigma)=0 ;
\end{gathered}
$$

it thus follows that

$$
I\left(\nu_{R}, \nu_{R}\right) \rightarrow I(\nu, \nu) \geq 0 .
$$

Assume now that $I(\nu, \nu)=0$. Then $I\left(\nu_{R}, \nu_{R}\right) \rightarrow 0$ so that we can find sequences $R_{k} \nearrow+\infty$ and $\epsilon_{k} \searrow 0$ with

$$
I\left(\psi_{\epsilon_{k}} * \nu_{R_{k}}, \psi_{\epsilon_{k}} * \nu_{R_{k}}\right) \rightarrow 0
$$

Since

$$
I\left(\psi_{\epsilon_{k}} * \nu_{R_{k}}, \psi_{\epsilon_{k}} * \nu_{R_{k}}\right)=d_{n} \int \frac{\left|\psi_{\epsilon_{k}} \widehat{\widehat{\nu}}_{R_{k}}(\xi)\right|^{2}}{|\xi|^{n}} d m(\xi),
$$

it follows that $\psi_{\epsilon_{k}} \widehat{*}_{R_{k}} \rightarrow 0$ in $S^{\prime}$. Hence $\psi_{\epsilon_{k}} * \nu_{R_{k}} \rightarrow 0$ in $S^{\prime}$ which implies $\nu=0$.

\section{REFERENCES}

1. E. Bedford and B. A. Taylor, Plurisubharmonic functions with logarithmic singularities. Annales de l'Institut Fourier (Grenoble) 38 (1988), 133-171.

2. U. Cegrell, J.-E. Fornaess and B. A. Taylor, personal communication.

3. W. K. Hayman, Subharmonic Functions, vol. 2, Academic Press (1989), London.

4. L. Hörmander, Notions of Convexity, Birkhäuser (1994), Boston.

5. M. Klimek, Pluripotential Theory, Oxford Univ. Press (1991), Oxford.

6. N. S. Landkof, Foundations of Modern Potential Theory, Springer (1972), Berlin.

DEPARTMENT OF MATHEMATICS UMEÅ UNIVERSITY S-901 87 UMEÅ SWEDEN
INSTITUTE OF MATHEMATICS JAGIELLONIAN UNIVERSITY REYMONTA 4 30-059 KRAKÓW POLAND
DEPARTMENT OF MATHEMATICS UNIVERSITY OF AUCKLAND PRIVATE BAG 92019 AUCKLAND NEW ZEALAND 